\title{
Hybrid TDOA/AOA Localization Algorithm in Non-line-of-sight Environments
}

\author{
Rongpei Ni, Hui Xiong, Luning Xu, Yanchun Xie \\ School of Electronic Science and Engineering, National University of Defense Technology, \\ Changsha, China \\ xf2010128185@163.com
}

\begin{abstract}
In reality, Non-line-of-sight(NLOS) error is the major factor which influences the positioning accuracy. NLOS identification and correction are the major techniques of reducing the positioning error. In this paper, we investigate the NLOS propagation identification and correction for time difference of arrival (TDOA) in wireless sensor networks. Based on the TDOA residual, an NLOS identification algorithm is proposed. when the anchors which can use to location are too small, after we identified. In this case, the excessive time delay in NLOS environment; the measurements for TDOA are reconstructed by estimating the mean and variance of the excessive time delay. So we can rewrite the TDOA that we measured. In order to obtain an accuracy position, we joint AOA to this location. In this case, we can calculate the tag position with TDOA and AOA. Firstly, it figures out the radial range between tag and reference anchor, then calculates the location of tag. Simulation results show that with the existence of NLOS errors, this method can improve the positioning accuracy in wireless sensor networks.
\end{abstract}

Keywords - NLOS error; identification ;time difference of arrival(TDOA); angle of arrival (AOA)

\section{INTRODUCTION}

Wireless location is important in Emergency 911 subscriber safety service and senor network applications, such as indoor navigation and surveillance [1]-[3].With the development of science and technology, more and more people focus their attention on this area for its potential application. The dominant anchor location techniques based on received signal strength (RSS), time of arrival (TOA), time difference of arrival (TDOA), angle of arrival (AOA), or combination of two of the above, can achieve high accuracy under the line-of-sight (LOS) propagation. And the actual environment is mostly NLOS environment, it is important to solve the problem of the NLOS. None-line-of-sight (NLOS) propagation introduces a bias in the TOA, TDOA, and AOA measurements and also results in an extra power loss. The performance of location techniques mentioned previously degrades evidently in NLOS scenarios. Therefore, it is significant to find methods to mitigate the NLOS bias.

In wireless sensor networks, the major error in time of arrival(TDOA) is measurement noise and non-line-ofsight(NLOS) propagation error .Measurement noise is usually considered as a Gaussian random variable, which mean is zero .However, NLOS error usually has an unknown distribution with a positive mean .NLOS error is the dominant

This work was supported by the National Natural Science Foundation of China (Grant No:61571452) error in location estimation[1].

In order to protect locations estimate from NLOS error corruption, NLOS identification and reconstruction techniques have been investigated. In general, NLOS range measurements have a larger variance than LOS range measurements, especially when the tag is moving. In [4], an NLOS base station (BS) is identified by calculating the standard deviation of a series of range measurements and comparing that with a certain threshold. A Kalman filter is then used with an extra unknown constant, the NLOS range error, to reconstruct the NLOS error and obtain the location estimate. A time-history based hypothesis test is proposed in [5] to identify and then to reconstruct the NLOS error. In [6], a decision theoretic framework for NLOS identification is formulated, where the NLOS error is modeled as a non-zero mean Gaussian random variable. If the stochastic model for the NLOS error is not Gaussian, the approach in [6] relies only on the fact that the variance of the NLOS errors is greater than that of the LOS errors.

Other papers are also showed another method to NLOS .In [7], a scattering model is used to solve the NLOS problem. In [8], they used the concept of virtual reference device to deal with the NLOS.

In this paper, we investigate the NLOS error and proposed an identification algorithm in indoor location by means of jointing TDOA and AOA location. The remainder of this paper is organized as follows. Section II presents the model of TOA and AOA measurements error. Section III presents the hybrid TDOA and AOA location. Section IV discusses the simulation results to show the advantages of the proposed strategies. Finally, the concluding remarks and future work are provided in Section V.

\section{SYSTEM MODEL}

Assuming that the network is fully connected that both the TOA and AOA measurements are available.

\section{A. major of TOA error}

The TOA measurement errors consist of noise and NLOS error, so the TOA measurement model can be considered as:

$$
\tau=\tau_{\text {los }}+\tau_{\text {noise }}+\tau_{\text {nlos }}
$$

Where the $\tau$ is the time of arrival, $\tau_{\text {los }}$ is the time of arrival when the signal is under line of sight propagation condition, 
$\tau_{\text {noise }}$ is an additive Gaussian random variable with zero mean and variance $\sigma_{\text {noise }}$, and $\tau_{\text {noise }}$ is the measurement error caused by the NLOS propagation.

\section{B. major of AOA error}

We consider the AOA suffers from the noise error, which it is modeled to be Gaussian random variable $N\left(0, \sigma_{\theta}\right)$, Then the AOA measurement model can be considered as:

$$
\theta=\theta^{0}+\theta_{\text {noise }}
$$

Where $\theta^{0}$ present the real AOA, $\sigma_{\text {noise }}$ present the measurement noise.

\section{NLOS identification}

Consider the NLOS error for TDOA and AOA location. The intersection points of these hyperbolas define an area of uncertainty where the final estimate of tag location lies in. For a given set $S_{k}$ of anchors and a reference tag location $(\hat{x}, y)$, we define the TDOA residual as the difference between the measured data and the calculated data using the reference location

$$
R E S_{k}=\sum_{B S_{i} \in S_{k}}\left[\left(\hat{d}_{i}-d_{1}\right)-c t_{i}\right]^{2}
$$

Where

$$
d_{i}=\sqrt{\left(x_{i}-\hat{x}\right)^{2}+\left(y_{i}-y\right)^{2}}
$$

is the ith anchor location.

The algorithm of identifying an NLOS anchor in TDOA location has the following steps:(a) Assuming there are $\mathrm{m}$ anchor nodes, we select $n$ (Number of anchor nodes required to locate) anchor nodes to find the location of tag. We have $C_{m}^{n}$ combinations.(b) From the first set of anchor, we can get the TDOA of each anchor, so we can estimate the location of $\operatorname{tag}(\hat{x}, y)$. (c) we can utilize (9)and (10) get $R E S_{1}$ By the same way, we can get the rest sets of data $R E S_{i}$ then calculate the total weight of each BS by summing the weights of the anchor sets that the anchor belongs to.(d) Rank each BS according to the total weight, and pick up the one with the heaviest weight.

\section{NLOS error correction}

From [13],If we consider the NLOS error obey exponential distribution. The probability density function can be expressed as:

$$
f\left(\tau_{\text {nlos }}\right)=\frac{1}{\tau_{\text {rms }}} \exp \left(-\frac{\tau_{\text {nlos }}}{\tau_{\text {rms }}}\right)
$$

Where $\tau_{r m s}$ is rms delay spread defined as [8]:

$$
\tau_{r m s}=T_{1} d^{\varepsilon} \zeta
$$

Where $T_{1}$ is median value of $r m s$ delay spread at one kilometer, $d$ is the distance between tag and anchor, $\varepsilon$ is an exponent with value between 0.5 and $1, \zeta$ is a lognormal variable with zero mean and standard deviation $\sigma_{\zeta}$ that lies between 4-6 dB $T_{1}$ Values of different channels are shown in Table 1:

TABLE I. $\quad T_{1}$ IN DIFFERENT ENVIRONMENTS

\begin{tabular}{|c|c|}
\hline Channel environment & $T_{1}(\mu \mathrm{S})$ \\
\hline downtown area & 1.0 \\
\hline General urban area & 0.4 \\
\hline suburb & 0.3 \\
\hline outskirts & 0.1 \\
\hline
\end{tabular}

From (2)we can know

$$
\ln \tau_{r m s}=\ln \left(T_{1} d^{\varepsilon}\right)+\ln \zeta
$$

Because $\ln \zeta \sim N\left(0, \sigma_{\zeta}^{2}\right)$, so we can get $\ln \tau_{r m s} \sim N\left(m_{\zeta}, \sigma_{\zeta}^{2}\right), m_{\zeta}=\ln \left(T_{1} d^{\varepsilon}\right)$, The probability density function of $\tau_{\text {rms }}$ :

$$
f\left(\tau_{r m s}\right)=\frac{1}{\sqrt{2 \pi} \sigma_{\zeta} \tau_{r m s}} \exp \left[-\frac{\left(\ln \tau_{r m s}-m_{\zeta}\right)^{2}}{2 \sigma_{\zeta}^{2}}\right]
$$

The mean and variance of the exponential distribution respectively is

$$
\begin{gathered}
\mu_{e}=E\left(\tau_{r m s}\right)=\int_{0}^{\infty} \tau_{r m s} f\left(\tau_{r m s}\right) d \tau_{r m s}=\exp \left(m_{i}+\frac{\sigma_{\zeta}^{2}}{2}\right) \\
\sigma_{e}^{2}=E\left(\tau_{r m s}^{2}\right)=\int_{0}^{\infty} \tau_{r m s}^{2} f\left(\tau_{r m s}\right) d \tau_{r m s}= \\
\exp \left(2 m_{\zeta}\right)\left(2 \exp \left(2 \sigma_{\zeta}^{2}\right)-\exp \left(\sigma_{\zeta}^{2}\right)\right)
\end{gathered}
$$

\section{HYBRID TDOA AND AOA LOCATION}

In a two-dimensional plane, time difference can determine a distance difference. We can use three anchors to location tag by TDOA location .However, when the joint TDOA and AOA location algorithms used, only two stations are enough to estimate the target position. Assume the coordinates of the anchor are $\left(x_{i}, y_{i}\right)$, the coordinates of the tag are $(x, y)$.Take the anchor $\left(x_{0}, y_{0}\right)$ as the reference anchor.

The relationship of the target relative to the reference anchor can be expressed as

$$
\tan \theta=\frac{y-y_{0}}{x-x_{0}}
$$

And the distance between tag and anchor $d_{i}$ is 


$$
d_{i}=\sqrt{\left(x-x_{i}\right)^{2}+\left(y-y_{i}\right)^{2}}
$$

So we can get the reference anchor between tag is

$$
d_{0}=\sqrt{\left(x-x_{0}\right)^{2}+\left(y-y_{0}\right)^{2}}
$$

The distance between 1st anchor and tag is

$$
d_{1}=\sqrt{\left(x-x_{1}\right)^{2}+\left(y-y_{1}\right)^{2}}
$$

The time difference we can express

$$
\Delta \tau=\frac{1}{c}\left(d_{i}-d_{0}\right)=\frac{\Delta d}{c}
$$

Which

$$
\Delta d=r_{1}-r_{0}
$$

Combine (13)(14)(16) we have

$$
\begin{aligned}
& d_{1}^{2}-d_{0}^{2}=\left(x-x_{1}\right)^{2}+\left(y-y_{1}\right)^{2}- \\
& \left(x-x_{0}\right)^{2}-\left(y-y_{0}\right)^{2}
\end{aligned}
$$

Since $\Delta d=d_{1}-d_{0}, d_{1}+d_{0}=2 d_{0}+\Delta d$ So

$$
\begin{aligned}
& \left(2 d_{0}+\Delta d\right) \Delta d=\left[2 x-\left(x_{1}+x_{0}\right)\right]\left(x_{0}-x_{1}\right) \\
& +\left[2 y-\left(y_{1}+y_{0}\right)\right]\left(y_{0}-y_{1}\right)
\end{aligned}
$$

Transform and arrange (19), we can obtain

$$
\begin{aligned}
& \left(x_{0}-x_{1}\right) x+\left(y_{0}-y_{1}\right) y=d_{0} \Delta d+ \\
& \frac{1}{2}\left[\Delta d^{2}+\left(x_{0}^{2}+y_{0}^{2}\right)-\left(x_{1}^{2}+y_{1}^{2}\right)\right]
\end{aligned}
$$

Let

$$
h=\frac{1}{2}\left[\Delta d^{2}+\left(x_{0}^{2}+y_{0}^{2}\right)-\left(x_{1}^{2}+y_{1}^{2}\right)\right]
$$

Equation (20) can be rewrite as

$$
\left(x_{0}-x_{1}\right) x+\left(y_{0}-y_{1}\right) y=h+d_{0} \Delta d
$$

From (3), we can get

$$
x \tan \theta-y=x_{0} \tan \theta-y_{0}
$$

Expressing (18) and (22) in a linear equation, we can have

$$
A x=b
$$

Where

$$
\begin{gathered}
A=\left[\begin{array}{cc}
x_{0}-x_{1} & y_{0}-y_{1} \\
\tan \theta & -1
\end{array}\right], x=\left[\begin{array}{l}
x \\
y
\end{array}\right] \\
b=\left[\begin{array}{c}
h+d_{0} \Delta d \\
x_{0} \tan \theta-y_{0}
\end{array}\right]
\end{gathered}
$$

Assuming $d_{0}$ is known, if the location of two anchor satisfy the expression $x_{0}-x_{1} \neq-\left(y_{0}-y_{1}\right) \tan \theta$, A have a reversible matrix, so the (22) can be expressed as

$$
x=A^{-1} b
$$

Let

$$
A^{-1}=\left[Z_{i j}\right]_{2 \times 2}
$$

We can obtain

$$
\begin{aligned}
& {\left[\begin{array}{l}
x \\
y
\end{array}\right]=\left[\begin{array}{ll}
z_{11} & z_{12} \\
z_{21} & z_{22}
\end{array}\right]\left[\begin{array}{c}
h+d_{0} \Delta d \\
x_{0} \tan \theta-y_{0}
\end{array}\right]=} \\
& {\left[\begin{array}{c}
z_{11}\left(h+d_{0} \Delta d\right)+z_{12}\left(x_{0} \tan \theta-y_{0}\right) \\
z_{21}\left(h+d_{0} \Delta d\right)+z_{22}\left(x_{0} \tan \theta-y_{0}\right)
\end{array}\right]=} \\
& {\left[\begin{array}{c}
z_{11} h+z_{12}\left(x_{0} \tan \theta-y_{0}\right)+z_{11} d_{0} \Delta d \\
z_{21} h+z_{22}\left(x_{0} \tan \theta-y_{0}\right)+z_{21} d_{0} \Delta d
\end{array}\right]}
\end{aligned}
$$

We can assume

$$
\left\{\begin{array}{c}
p_{i}=z_{i 1} \Delta d \\
q_{i}=z_{i 1} h+z_{i 2}\left(x_{0} \tan \theta-y_{0}\right)
\end{array} i=1,2,\right.
$$

then we can get

$$
\left[\begin{array}{l}
x \\
y
\end{array}\right]=\left[\begin{array}{c}
q_{1}+p_{1} d_{0} \\
q_{2}+p_{2} d_{0}
\end{array}\right]
$$

Combine (13) and (29)

We can get

$$
a d_{0}^{2}+2 b d_{0}+c=0
$$

Where

$$
\left\{\begin{array}{c}
a=p_{1}^{2}+p_{2}^{2}-1 \\
b=\left(q_{1}-x_{0}\right) p_{1}+\left(q_{2}-y_{0}\right) p_{2} \\
c=\left(q_{1}-x_{0}\right)^{2}+\left(q_{2}-y_{0}\right)^{2}
\end{array}\right.
$$

From (30) and (31) we can get the value of $d_{0}$, and then we can get the value of the $\mathrm{x}(x, y)$ which is the location of tag.

However this algorithm has limitations.

If $\Delta=b^{2}-4 a c<0$, we cannot get the value of $d_{0}$ we cannot obtain the location of tag.

If $\Delta=b^{2}-4 a c \geq 0$, we can get the value of $d_{0}=\frac{-b \pm \sqrt{b^{2}-a c}}{a}$, and when $\Delta=b^{2}-4 a c=0$, we can get only one value, if $d_{0}>0$, we can get the location of the tag, if $d_{0}>0$, the location cannot be got. When $\Delta=b^{2}-4 a c>0$, we will have two values, if two value are bigger than zero, we can obtain two value, it is necessary to eliminate the uncertain value. If the two values are smaller than zero, we cannot obtain 
the tag location. If one value is bigger than zero, another is smaller than zero; we can get a exact value of the tag location.

\section{Simulation RESULTS}

Simulation is set in a two-dimensional space, there are 4 anchor nodes and 1 Tag as shown in Figure 2

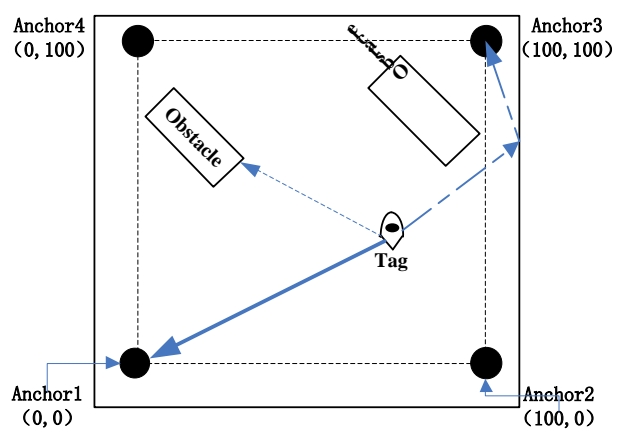

Fig. 1. Simulation scene

This part of the simulation analysis in the same number of non line of sight, the average value of different NLOS error, the improved differential compensation algorithm for positioning performance.

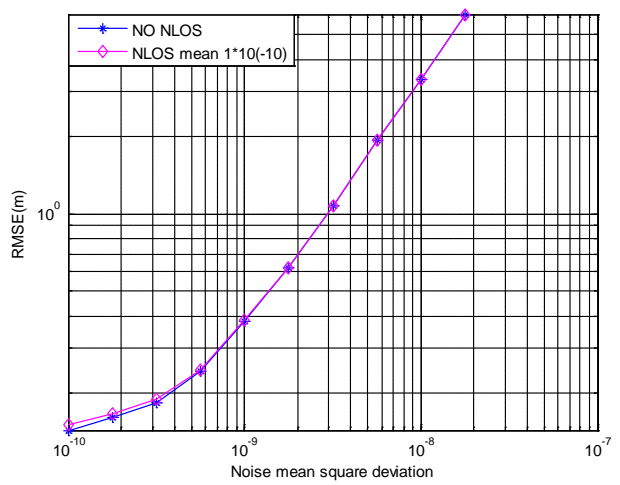

Fig. 2. Effect of NLOS on RMSE

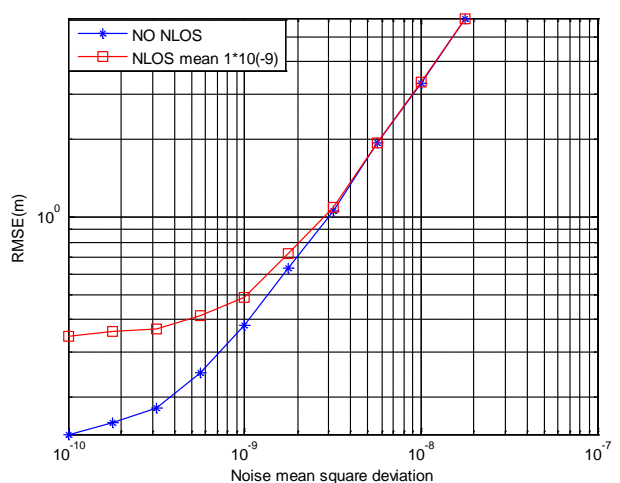

Fig. 3. Effect of NLOS on RMSE

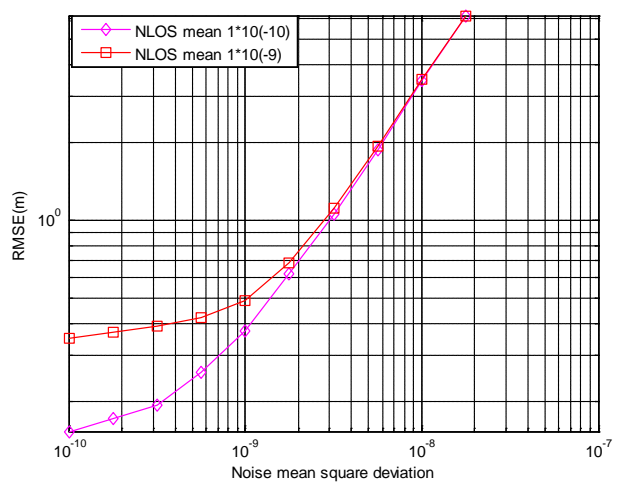

Fig. 4. Effects of different NLOS on RMSE

These figures show the influence of NLOS error which mean is $10^{-9}$ or $10^{-10}$ on the positioning accuracy. In the algorithm, if we find the anchor in NLOS, the NLOS anchor will be removed do not participate in the position. It can be seen from the figure that the hybrid TDOA and AOA algorithm positioning accuracy, and the influence of NLOS error with different mean. We can get the algorithm have a positive effect of the tag location in NLOS environment. However, when the noise mean square deviation is $10^{-7}$, we cannot get a value, for the limit of this hybrid TDOA and AOA algorithm positioning, as the above analysis.

\section{CONCLUSIONS}

In this paper, a NLOS identification algorithm and the NLOS error correction for TDOA is proposed. The NLOS identification is based on the TDOA residual. The exponential distribution model is used to NLOS error. And this paper combines with a known hybrid TDOA and AOA location algorithm shows that the effect of NLOS error on the positioning accuracy. From the simulation results we can get that the proposed algorithm is feasible.

\section{References}

[1] G. Seco-Grandados, J. A. Lopez-Salcedo, D. Jimenez-Banos, and G.Lopez-Risueno, "Challenges in indoor global navigation satellite systems:Unveiling its core features in signal processing,” IEEE Signal Process. Mag., vol. 29, no. 2, pp. 108-131, Mar. 2012.

[2] Y. Jin, W.-S. Soh, M. Motani, and W.-C. Wong, "A robust indoor pedestrian tracking system with sparse infrastructure support,” IEEE Trans. Mobile Comput., vol. 12, no. 7, pp. 1392-1403, Jul. 2013.

[3] P. Bahl and V. Padmanabhan, "RADAR: An in-building RF based user location and tracking system,” in Proc. IEEE INFOCOM, pp. 775-784, 2000 .

[4] S.-S. Woo, H.-R. You, and J.-S. Koh, "The NLOS mitigation technique for position location using IS-95 CDMA networks,” in Proc. IEEE VTC,vol. 4, pp. 2556-2560, September 2000.

[5] M. P. Wylie and J. Holtzman, “The non-line of sight problem in mobile location estimation," in Proc. IEEE International Conference on Universal Personal Communications, vol. 2, pp. 827-831, 1996.

[6] J. Borr`as, P. Hatrack, and N. B. Mandayam, "Decision theoretic framework for NLOS identification,” in Proc. IEEE VTC, vol. 2, pp. 1583-1587, 1998.

[7] Al-Jazzar, Saleh, James Caffery Jr, and Heung-Ryeol You. "Scatteringmodel-based methods for TOA location in NLOS 
environments."Vehicular Technology, IEEE Transactions on 56.2: 583$593,2007$.

[8] L. J. Greenstein, V. Erecg, Y. S. Yeh, and M. V. Clark, “A New PathGain Delay-Spread Propagation Model for Digital Cellular Channels,”Vehicular Technology, IEEE Transactions on, vol. 46, no. 2, pp. 477-485, 1997.

[9] Xiong H, Chen Z, An W, et al. "Robust TDOA localization algorithm for asynchronous wireless sensor networks."International Journal of Distributed Sensor Networks2015,Oct,2015.

[10] Hui, X., Zhiyuan, C., Beiya, Y., \& Rongpei, N. "TDOA localization algorithm with compensation of clock offset for wireless sensor networks."Communications, China,pp. 193-201 Nov. 2015.

[11] Liu, Congfeng, Jie Yang, and Fengshuai Wang. "Joint TDOA and AOA location algorithm."Systems Engineering and Electronics, Journal of 24.2 pp.183-188. 2013..

[12] Cong, Li, and Weihua Zhuang. "Non-line-of-sight error mitigation in TDOA mobile location."Global Telecommunications Conference, 2001. GLOBECOM'01. IEEE. Vol. 1. IEEE, 2001.
[13] Greenstein, L. J., Erceg, V., Yeh, Y. S., \& Clark, M. V. "A new pathgain/delay-spread propagation model for digital cellular channels."Vehicular Technology, IEEE Transactions on 46.2 (1997): 477-485.

[14] Ding, G., Tan, Z., Zhang, L., Zhang, Z., \& Zhang, J. "Hybrid TOA/AOA cooperative localization in non-line-of-sight environments."Vehicular Technology Conference (VTC Spring), 2012 IEEE 75th. IEEE, 2012.

[15] Chen, S. W., C. K. Seow, and S. Y. Tan. "Virtual reference device-based NLOS localization in multipath environment."Antennas and Wireless Propagation Letters, IEEE 13 (2014): 1409-1412.

[16] Chen, Pi-Chun. "A non-line-of-sight error mitigation algorithm in location estimation."Wireless Communications and Networking Conference, 1999. WCNC. IEEE, 1999.

[17] Wylie, Marilynn P., and Jack Holtzman. "The non-line of sight problem in mobile location estimation."Universal Personal Communications, 1996. Record., 1996 5th IEEE International Conference on. Vol. 2. IEEE, 1996. 